\title{
Pointwise Trichotomy For Skew-Evolution Semiflows on BanaCh SPACES
}

\author{
Codruţa Stoica
}

\begin{abstract}
The paper introduces the notion of skew-evolution semiflows and presents the concept of pointwise trichotomy in the case of skew-evolution semiflows on a Banach space. The connection with the classic notion of trichotomy presented in [8], for evolution operators, is also emphasized, as well as some characterizations. The approach of the theory is from uniform point of view. The study can also be extended to systems with control whose state evolution can be described by skew-evolution semiflows.
\end{abstract}

Mathematics Subject Classification: 34D09

Keywords: Skew-evolution semiflow, exponential trichotomy, pointwise exponential trichotomy

\section{Introduction}

It is in [1] that S.N. Chow and H. Leiva introduced and characterized the notion of pointwise discrete dichotomy for discrete skew-product flows. The existence of this asymptotic property is related to the existence of a unique bounded solution of certain difference equation, involving the discrete cocycle. They also established the connection between the latest concept and the classical uniform exponential dichotomy for linear skew-product flows.

In [3] Y. Latushkin, S. Montgomery-Smith and T. Randolph studied the spectral mapping and the hyperbolicity, related to the exponential dichotomy, for evolutionary semigroups generated by a strongly continuous semicocycle over a locally compact metric space acting on Banach fibers. The results are applied in the study of pointwise dichotomy versus global dichotomy. Later, in [4] is studied the exponential dichotomy of an exponentially bounded, strongly continuous cocycle on a locally compact metric space acting on a Banach space. Dichotomy is characterized in terms of hyperbolicity of a family of weighted shift operators. It is shown that exponential dichotomy follows from pointwise discrete dichotomies with uniform constants.

In [7] M. Megan, A.L. Sasu and B. Sasu emphasize the concept of pointwise exponential dichotomy for linear skew-product flows, by neces- 
sary and sufficient conditions, as well as the relation with global exponential dichotomy. It is proved that the presented property for skew-product flows is equivalent with the pointwise admissibility of the pair $\left(C_{0}(\mathbb{R}, \mathcal{V}), C_{0}(\mathbb{R}, \mathcal{V})\right)$, $\mathcal{V}$ being a Banach space.

Systems with control are studied in [5], their state evolution being described by linear skew-product semiflows. It is also presented the relationship between the concepts of exact controllability and complete stabilizability of general control systems.

The concept of trichotomy is considered as a natural generalization of the classic concept of dichotomy. The basic idea is to obtain, at any moment, a splitting of the space into three subspaces: the stable subspace, the unstable subspace and the neutral one. The notion of Sacker-Sell type trichotomy was introduced in 1976 and studied in the case of linear differential equations in [11]. For the first time a sufficient condition for the existence of trichotomy in the case of $\mathbb{R}^{n}$ was emphasized.

Later, in 1988, a stronger notion was introduced by S. Elaydi and O. Hajek in [2], namely the exponential trichotomy for linear and nonlinear differential systems by means of Liapunov functions.

In [8] we have presented the property of uniform exponential trichotomy in the case of evolution operators in Banach spaces. The results extend wellknown theorems obtained in the linear case for uniform exponential stability, but we emphasize a unified treatment for uniform asymptotic behaviors (exponential decay, exponential growth, exponential stability, exponential instability, exponential dichotomy, exponential trichotomy, as given in [12]) in the setting of nonlinear evolution operators.

Equivalent definitions and characterizations for the uniform exponential trichotomy by means of two respectively four compatible projector families are given in 9]. Some properties for a particular case of skew-evolution semiflows were introduced in [10].

The theory of skew-evolution semiflows introduced by us is used to study the asymptotic behavior of time-varying linear systems, the question that arises being whether the connection between stabilizability and controllability can be extended to systems associated to skew-evolution semiflows.

In our present paper we extend the study of exponential trichotomy in a more general setting fitting the new concept of skew-evolution semiflows, from a global and a pointwise point of view, some similarities and differences being emphasized. The study of the concept of skew-evolution semiflow for the approach of evolution equations by means of the evolution operators theory was essential. 


\section{Definitions and examples}

Let $(\mathcal{X}, d)$ be a metric space, $\mathcal{V}$ a Banach space, $\mathcal{B}(\mathcal{V})$ the space of all $\mathcal{V}$ valued bounded operators defined on $\mathcal{V}$. We denote $\mathcal{Y}=\mathcal{X} \times \mathcal{V}$ and we consider the set $\mathcal{T}=\left\{\left(t, t_{0}\right) \in \mathbb{R}_{+}^{2}: t \geq t_{0}\right\}$. The norm of vectors on $\mathcal{V}$ and operators on $\mathcal{B}(\mathcal{V})$ is denoted by $\|\cdot\|$. Let $I$ be the identity operator on $\mathcal{V}$.

Definition 2.1 A mapping $\psi: \mathcal{T} \times \mathcal{X} \rightarrow \mathcal{X}$ is called evolution semiflow on $\mathcal{X}$ if it satisfies the following properties

$$
\begin{aligned}
& \left(e s_{1}\right) \psi(t, t, x)=x, \forall(t, x) \in \mathbb{R}_{+} \times \mathcal{X} \\
& \left(e s_{2}\right) \psi\left(t, s, \psi\left(s, t_{0}, x\right)\right)=\psi\left(t, t_{0}, x\right), \forall(t, s),\left(s, t_{0}\right) \in \mathcal{T}, \forall x \in \mathcal{X} .
\end{aligned}
$$

Definition 2.2 A mapping $\Psi: \mathcal{T} \times \mathcal{X} \rightarrow \mathcal{B}(\mathcal{V})$ that satisfies the following properties

$\left(e c_{1}\right) \Psi(t, t, x)=I, \forall t \geq 0, \forall x \in \mathcal{X}$

$\left(e c_{2}\right) \Psi\left(t, t_{0}, x\right)=\Psi\left(t, s, \psi\left(s, t_{0}, x\right)\right) \Psi\left(s, t_{0}, x\right), \forall(t, s),\left(s, t_{0}\right) \in \mathcal{T}, \forall x \in \mathcal{X}$ is called evolution cocycle over the evolution semiflow $\psi$.

Definition 2.3 A function $\xi: \mathcal{T} \times \mathcal{Y} \rightarrow \mathcal{Y}$ defined by

$$
\xi(t, s, x, v)=(\psi(t, s, x), \Psi(t, s, x) v), \forall(t, s, x, v) \in \mathcal{T} \times \mathcal{Y}
$$

where $\Psi$ is an evolution cocycle over the evolution semiflow $\psi$, is called skew-evolution semiflow on $\mathcal{Y}$.

Example 2.1 Let $f: \mathbb{R} \rightarrow \mathbb{R}_{+}$be a function which is nondecreasing on the interval $(-\infty, 0)$ and decreasing on the interval $(0, \infty)$ with the property that there exists

$$
\lim _{t \rightarrow \pm \infty} f(t) \in(0, \infty)
$$

Let us consider the set $\mathcal{C}=\mathcal{C}(\mathbb{R}, \mathbb{R})$ of all continuous functions given by $x: \mathbb{R} \rightarrow \mathbb{R}$, endowed with the uniform convergence topology on compact subsets of $\mathbb{R}$. $\mathcal{C}$ is metrizable by respect to the metric

$$
d(x, y)=\sum_{n=1}^{\infty} \frac{1}{2^{n}} \frac{d_{n}(x, y)}{1+d_{n}(x, y)}
$$

where

$$
d_{n}(x, y)=\sup _{t \in[-n, n]}|x(t)-y(t)| .
$$

Let $\mathcal{X}$ be the closure in $\mathcal{C}$ of the set $\left\{f_{t}, t \in \mathbb{R}\right\}$, where

$$
f_{t}(\tau)=f(t+\tau), \forall \tau \in \mathbb{R}, f \in \mathcal{C} .
$$

Then $(\mathcal{X}, d)$ is a metric space and the mapping

$$
\psi: \mathcal{T} \times \mathcal{X} \rightarrow \mathcal{X}, \psi(t, s, x)=x_{t-s}
$$


is an evolution semiflow on $\mathcal{X}$.

The mapping

$$
\Psi: \mathcal{T} \times \mathcal{X} \rightarrow \mathfrak{B}(\mathcal{V})
$$

given by

$$
\Psi(t, s, x)(v)=e^{\int_{s}^{t} x(\tau-s) d \tau} v
$$

is an evolution cocycle.

Then $\xi=(\psi, \Psi)$ is a skew-evolution semiflow on $\mathcal{Y}$.

Definition 2.4 A mapping $P: \mathcal{Y} \rightarrow \mathcal{Y}$ is said to be a projector on $\mathcal{Y}$ if $P$ is continuous and has the form

$$
P(x, v)=(x, P(x) v), \quad(x, v) \in \mathcal{Y}
$$

where $P(x)$ is a projection on $\mathcal{Y}_{x}=\{x\} \times \mathcal{V}, x \in \mathcal{X}$.

Remark 2.1 The function $P(x): \mathcal{Y}_{x} \rightarrow \mathcal{Y}_{x}$ is a bounded mapping with the property $P(x) P(x)=P^{2}(x)=P(x)$ for all $x \in \mathcal{X}$.

Definition 2.5 The mapping $Q: \mathcal{Y} \rightarrow \mathcal{Y}$ given by

$$
Q(x, v)=(x, v-P(x) v)
$$

where $P$ is a projector on $\mathcal{Y}$, is called complementary projector to $P$ on $\mathcal{Y}$.

Definition 2.6 A projector $P$ on $\mathcal{Y}$ is said to be invariant on $\mathcal{Y}$ relative to a skew-evolution semiflow $\xi=(\psi, \Psi)$ if one has

$$
P(\psi(t, s, x)) \Psi(t, s, x) v=\Psi(t, s, x) P(x) v,
$$

for all $(t, s) \in \mathcal{T}$ and for all $(x, v) \in \mathcal{Y}$, where $\Psi$ denotes an evolution cocycle over the semiflow $\psi$.

Remark 2.2 If the projector $P$ is invariant, then the complementary projector $Q$ is invariant as well.

Let us denote $\Psi_{k}\left(t, t_{0}, x\right)=\Psi\left(t, t_{0}, x\right) P_{k}(x)$, for all $\left(t, t_{0}\right) \in \mathcal{T}$, all $x \in \mathcal{X}$ and $k \in\{0,1,2\}$.

Definition 2.7 Three projector families $\left\{P_{k}(y)\right\}_{y \in \mathcal{Y}}, k \in\{0,1,2\}$, are said to be compatible with a skew-evolution semiflow $\xi$ if

$\left(c_{1}\right)$ each of the projectors $P_{k}, k \in\{0,1,2\}$ is invariant on $\mathcal{Y}$

$\left(c_{2}\right)$ for each $x \in \mathcal{X}$ and for all $i, j \in\{0,1,2\}, i \neq j$

$$
P_{0}(x)+P_{1}(x)+P_{2}(x)=I \text { and } P_{i}(x) P_{j}(x)=0 .
$$


Definition 2.8 A skew-evolution semiflow $\xi=(\psi, \Psi)$ has uniform exponential trichotomy on $\mathcal{Y}$ if there exist $N_{0}, N_{1}, N_{2}>1, \nu_{0}, \nu_{1}, \nu_{2}>0$ and three projector families $\left\{P_{k}(y)\right\}_{y \in \mathcal{Y}}, k \in\{0,1,2\}$, compatible with $\xi$ such that

$\left(t_{0}\right)$

$$
\left\|P_{0}(x) v\right\| \leq N_{0} e^{\nu_{0}\left(t-t_{0}\right)}\left\|\Psi_{0}\left(t, t_{0}, x\right) v\right\| \leq N_{0}^{2} e^{2 \nu_{0}\left(t-t_{0}\right)}\left\|P_{0}(x) v\right\|
$$

$\left(t_{1}\right)$

$\left(t_{2}\right)$

$$
e^{\nu_{1}\left(t-t_{0}\right)}\left\|\Psi_{1}\left(t, t_{0}, x\right) v\right\| \leq N_{1}\left\|P_{1}(x) v\right\|
$$

$$
e^{\nu_{2}\left(t-t_{0}\right)}\left\|P_{2}(x) v\right\| \leq N_{2}\left\|\Psi_{2}\left(t, t_{0}, x\right) v\right\|
$$

for all $\left(t, t_{0}\right) \in \mathcal{T}$ and all $(x, v) \in \mathcal{Y}$.

Remark 2.3 $(i)$ For $P_{0}=0$ in Definition 2.8 we obtain the property of uniform exponential dichotomy.

(ii) If $P_{0}=P_{2}=0$ the property of uniform exponential stability is obtained. It follows that a uniformly exponentially stable skew-evolution semiflow is uniformly exponentially dichotomic and, further, uniformly exponentially trichotomic.

(iii) Also it is easy to observe that the property of uniform exponential instability implies the uniform exponential dichotomy and, further, the uniform exponential trichotomy.

Example 2.2 Let $f: \mathbb{R}_{+} \rightarrow(0, \infty)$ be a decreasing function with the property that there exists $\lim _{t \rightarrow \infty} f(t)=l>0$. Let $\mu>f(0)>0$.

Let $(\mathcal{X}, d)$ be the metric space given as in Example 2.1, the closure in $\mathcal{C}(\mathbb{R}, \mathbb{R})$ of the set $\left\{f_{t}, t \in \mathbb{R}\right\}$, where

$$
f_{t}(\tau)=f(t+\tau), \forall \tau \in \mathbb{R}, f \in \mathcal{C} .
$$

The mapping

$$
\psi: \mathcal{T} \times \mathcal{X} \rightarrow \mathcal{X}, \psi(t, s, x)=x_{t-s},
$$

is an evolution semiflow on $\mathcal{X}$.

We consider $\mathcal{V}=\mathbb{R}^{3}$ with the norm

$$
\|v\|=\left|v_{1}\right|+\left|v_{2}\right|+\left|v_{3}\right|, v=\left(v_{1}, v_{2}, v_{3}\right) \in \mathbb{R}^{3} .
$$

The mapping $\Psi: \mathcal{T} \times \mathcal{X} \rightarrow \mathcal{B}\left(\mathbb{R}^{3}\right)$ defined by

$$
\begin{gathered}
\Psi\left(t, t_{0}, x\right) v= \\
=\left(e^{-\mu\left(t-t_{0}\right)+\int_{t_{0}}^{t} x\left(\tau-t_{0}\right) d \tau} v_{1}, e^{\int_{t_{0}}^{t} x\left(\tau-t_{0}\right) d \tau} v_{2}, e^{-\left(t-t_{0}\right) x(0)+\int_{t_{0}}^{t} x\left(\tau-t_{0}\right) d \tau} v_{3}\right),
\end{gathered}
$$

for all $\left(t, t_{0}\right) \in \mathcal{T}$ and all $(x, v) \in \mathcal{Y}$, is an evolution cocycle. 
We consider the projections given by

$$
P_{1}(x)(v)=\left(v_{1}, 0,0\right), P_{2}(x)(v)=\left(0, v_{2}, 0\right), P_{0}(x)(v)=\left(0,0, v_{3}\right)
$$

for all $x \in \mathcal{X}$ and all $v=\left(v_{1}, v_{2}, v_{3}\right) \in \mathcal{V}$.

We obtain the relations

$$
\begin{gathered}
\left.\left.\| \Psi\left(t, t_{0}, x\right) P_{1}(x) v\right)\left\|\leq e^{[-\mu+x(0)](t-s)}\right\| \Psi\left(s, t_{0}, x\right) P_{1}(x) v\right) \| \\
\left.\left.\| \Psi\left(t, t_{0}, x\right) P_{2}(x) v\right)\left\|\geq e^{l(t-s)}\right\| \Psi\left(s, t_{0}, x\right) P_{2}(x) v\right) \| \\
\left.\left.\| \Psi\left(t, t_{0}, x\right) P_{0}(x) v\right)\left\|\leq e^{x(0)(t-s)}\right\| \Psi\left(s, t_{0}, x\right) P_{0}(x) v\right) \| \\
\left.\left.\| \Psi\left(t, t_{0}, x\right) P_{0}(x) v\right)\left\|\geq e^{-x(0)(t-s)}\right\| \Psi\left(s, t_{0}, x\right) P_{0}(x) v\right) \|
\end{gathered}
$$

for all $(t, s),\left(s, t_{0}\right) \in \mathcal{T}$ and all $(x, v) \in \mathcal{Y}$.

It follows that the skew-evolution semiflow given by $\xi=(\psi, \Psi)$ is uniformly exponentially trichotomic with characteristics

$$
N_{0}=N_{1}=N_{2}=1 \text { and } \nu_{0}=x(0), \nu_{1}=-\mu+x(0), \nu_{2}=l .
$$

In what follows by projections we will consider the mappings $\widetilde{P}: \mathcal{Y} \rightarrow \mathcal{Y}$ with the property $\widetilde{P}^{2}(t)=\widetilde{P}(t), \forall t \geq 0$.

Definition 2.9 A family of projections $\{\widetilde{P}(t)\}_{t \geq 0}$ is said to be invariant on $\mathcal{Y}$ relative to a skew-evolution semiflow $\xi=(\psi, \Psi)$ if one has

$$
\widetilde{P}(t+s) \Psi(t, s, \psi(t, s, x)) v=\Psi(t, s, \psi(t, s, x)) \widetilde{P}(s) v,
$$

for all $(t, s) \in \mathcal{T}$ and for all $(x, v) \in \mathcal{Y}$, where $\Psi$ denotes an evolution cocycle over the semiflow $\psi$.

Definition 2.10 Three projection families $\left\{\widetilde{P}_{k}(t)\right\}_{t \geq 0}, k \in\{0,1,2\}$, are said to be compatible with a skew-evolution semiflow $\xi$ at point $x \in \mathcal{X}$ if

$\left(c p_{1}\right)$ each projection $\widetilde{P}_{k}, k \in\{0,1,2\}$ is invariant on $\mathcal{Y}$

$\left(c p_{2}\right)$ for each $t \in \mathbb{R}_{+}$one has

$$
\widetilde{P}_{0}(t)+\widetilde{P}_{1}(t)+\widetilde{P}_{2}(t)=I \text { and } \widetilde{P}_{i}(t) \widetilde{P}_{j}(t)=0, \forall i, j \in\{0,1,2\}, i \neq j
$$

$\left(c p_{3}\right)$ for all $t \geq 0$, all $v \in \mathcal{V}$ and all $i, j \in\{0,1,2\}, i \neq j$

$$
\left\|\widetilde{P}_{i}(t) v+\widetilde{P}_{j}(t) v\right\|^{2}=\left\|\widetilde{P}_{i}(t) v\right\|^{2}+\left\|\widetilde{P}_{j}(t) v\right\|^{2} .
$$

Definition 2.11 A skew-evolution semiflow $\xi=(\psi, \Psi)$ has pointwise uniform exponential trichotomy on $\mathcal{Y}$ at point $x_{0} \in \mathcal{X}$ if there exist some constants $N_{0}^{x_{0}}, N_{1}^{x_{0}}, N_{2}^{x_{0}}>1, \nu_{0}^{x_{0}}, \nu_{1}^{x_{0}}, \nu_{2}^{x_{0}}>0$ and three projection families $\left\{\widetilde{P}_{k}(t)\right\}_{t \geq 0}, k \in\{0,1,2\}$, compatible with $\xi$ such that 
$\left(p t_{0}\right)$

$$
\begin{gathered}
\| \Psi\left(s, t_{0}, \psi\left(s, t_{0}, x_{0}\right) \widetilde{P}_{0}\left(t_{0}\right) v \| \leq\right. \\
\leq N_{0}^{x_{0}} e^{\nu_{0}^{x_{0}}(t-s)}\left\|\Psi\left(t, t_{0}, \psi\left(t, t_{0}, x_{0}\right)\right) \widetilde{P}_{0}\left(t_{0}\right) v\right\| \\
\left\|\Psi\left(t, t_{0}, \psi\left(t, t_{0}, x_{0}\right)\right) \widetilde{P}_{0}\left(t_{0}\right) v\right\| \leq \\
\leq N_{0}^{x_{0}} e^{\nu_{0}^{x_{0}}(t-s)}\left\|\Psi\left(s, t_{0}, \psi\left(s, t_{0}, x_{0}\right)\right) \widetilde{P}_{0}\left(t_{0}\right) v\right\|
\end{gathered}
$$

$\left(p t_{1}\right)$

$$
\begin{gathered}
e^{\nu_{1}^{x_{0}}(t-s)}\left\|\Psi\left(t, t_{0}, \psi\left(t, t_{0}, x_{0}\right)\right) \widetilde{P}_{1}\left(t_{0}\right) v\right\| \leq \\
\leq N_{1}^{x_{0}}\left\|\Psi\left(s, t_{0}, \psi\left(s, t_{0}, x_{0}\right)\right) \widetilde{P}_{1}\left(t_{0}\right) v\right\|
\end{gathered}
$$

$\left(p t_{2}\right)$

$$
\begin{gathered}
e^{\nu_{2}^{x_{0}}(t-s)}\left\|\Psi\left(s, t_{0}, \psi\left(s, t_{0}, x_{0}\right)\right) \widetilde{P}_{2}\left(t_{0}\right) v\right\| \leq \\
\leq N_{2}^{x_{0}}\left\|\Psi\left(t, t_{0}, \psi\left(t, t_{0}, x_{0}\right)\right) \widetilde{P}_{2}\left(t_{0}\right) v\right\|
\end{gathered}
$$

for all $(t, s),\left(s, t_{0}\right) \in \mathcal{T}$ and all $v \in \mathcal{V}$.

Remark 2.4 According to [3] the concept introduced in Definition 2.11 can also be called exponential trichotomy of the skew-evolution semiflow $\xi$ over the orbit through $x_{0} \in \mathcal{X}$.

Remark 2.5 Referring to the case of pointwise dichotomy, in [3] and [1] is shown that for an invertible-valued cocycle involved in the definition of a skew-product flow, the later property is equivalent with the classical definition of global dichotomy.

We remark that the property of uniform exponential trichotomy for a skew-evolution semiflow implies the uniform exponential trichotomy at each point $x \in \mathcal{X}$, by considering $\widetilde{P}_{k}(t)=P_{k}(\psi(t, x)), \forall t \geq 0, k \in\{0,1,2\}$.

Instead, in general, the converse affirmation is not always true, as shown in the following

Example 2.3 Let $\mathcal{C}\left(\mathbb{R}_{+}, \mathbb{R}\right)=\left\{f: \mathbb{R}_{+} \rightarrow \mathbb{R} \mid f\right.$ continuous $\}$, metrizable with respect to the metric considered in Example 2.1.

For every $n \in \mathbb{N}^{*}$ we consider a decreasing function

$$
x_{n}: \mathbb{R}_{+} \rightarrow\left(\frac{1}{2 n+1}, \frac{1}{2 n}\right), \lim _{t \rightarrow \infty} x_{n}(t)=\frac{1}{2 n+1} .
$$

If $x_{n}^{s}(t)=x_{n}(t+s), \forall t, s \geq 0$ and $\mathcal{X}=\overline{\left\{x_{n}^{s}, n \in \mathbb{N}^{*}, s \in \mathbb{R}_{+}\right\}}$then the mapping

$$
\psi: \mathcal{T} \times \mathcal{X} \rightarrow \mathcal{X}, \psi(t, s, x)(\tau)=x(t-s+\tau)
$$


is an evolution semiflow on $\mathcal{X}$.

If we consider $\mathcal{V}=\mathbb{R}^{3}$ with the norm $\left\|\left(v_{1}, v_{2}, v_{3}\right)\right\|=\left|v_{1}\right|+\left|v_{2}\right|+\left|v_{3}\right|$, then $\xi=(\psi, \Psi)$, where $\Psi: \mathcal{T} \times \mathcal{X} \rightarrow \mathfrak{B}(\mathcal{V})$ is given by

$$
\Psi\left(t, t_{0}, x\right)\left(v_{1}, v_{2}, v_{3}\right)=\left(e^{-\int_{t_{0}}^{t} x(s) d s} v_{1}, e^{\int_{t_{0}}^{t} x(s) d s} v_{2}, e^{\int_{t_{0}}^{t} x(s) d s} v_{3}\right),
$$

is a skew-evolution semiflow on $\mathcal{Y}$. We consider the projections $P_{k}, k \in$ $\{0,1,2\}$ given as in Example 2.2. We obtain that $\xi$ is not globally uniformly exponentially trichotomic, but is uniformly exponentially trichotomic at every point $x \in \mathcal{X}$ relative to the projection families $\left\{\widetilde{P}_{k}(x)\right\}_{x \in \mathcal{X}}$, given by $\widetilde{P}_{k}(x)=P_{k}, x \in \mathcal{X}, k \in\{0,1,2\}$.

\section{Characterizations of the pointwise trichotomy}

Let us consider the set of functions

$$
\mathcal{F}=\left\{f:[0, \infty) \rightarrow(0, \infty) \mid f \text { decreasing function, } \lim _{t \rightarrow \infty} f(t)=0\right\}
$$

In order to emphasize the natural extension of the trichotomy relative to the property of dichotomy, we will present a characterization by means of two projection families compatible with a skew-evolution semiflow, introduced by the following

Definition 3.1 Two projection families $\left\{\widetilde{Q}_{k}(t)\right\}_{t \geq 0}$ where $\widetilde{Q}_{k}: \mathbb{R}_{+} \rightarrow \mathfrak{B}(\mathcal{V})$, $k \in\{1,2\}$, are said to be compatible with a skew-evolution semiflow $\xi$ at point $x \in \mathcal{X}$ if following relations hold

$$
\begin{aligned}
& \left(c q_{1}\right) \widetilde{Q}_{1}(t) \widetilde{Q}_{2}(t)=\widetilde{Q}_{2}(t) \widetilde{Q}_{1}(t)=0 \\
& \left(c q_{2}\right)\left\|\left[\widetilde{Q}_{1}(t)+\widetilde{Q}_{2}(t)\right] v\right\|^{2}=\left\|\widetilde{Q}_{1}(t) v\right\|^{2}+\left\|\widetilde{Q}_{2}(t) v\right\|^{2} \\
& \left(c q_{3}\right)\left\|\left[I-\widetilde{Q}_{1}(t)\right] v\right\|^{2}=\left\|\left[I-\widetilde{Q}_{1}(t)-\widetilde{Q}_{2}(t)\right] v\right\|^{2}+\left\|\widetilde{Q}_{2}(t) v\right\|^{2} \\
& \left(c q_{4}\right)\left\|\left[I-\widetilde{Q}_{2}(t)\right] v\right\|^{2}=\left\|\left[I-\widetilde{Q}_{1}(t)-\widetilde{Q}_{2}(t)\right] v\right\|^{2}+\left\|\widetilde{Q}_{1}(t) v\right\|^{2} \\
& \left(c q_{5}\right) \Psi\left(t, t_{0}, \psi\left(t, t_{0}, x\right)\right) \widetilde{Q}_{k}\left(t_{0}\right) v=\widetilde{Q}_{k}\left(t+t_{0}\right) \Psi\left(t, t_{0}, \psi\left(t, t_{0}, x\right)\right) v
\end{aligned}
$$

for all $\left(t, t_{0}\right) \in \mathcal{T}$, all $v \in \mathcal{V}$ and $k \in\{1,2\}$.

Theorem 3.1 A skew-evolution semiflow $\xi=(\psi, \Psi)$ is pointwise uniformly exponentially trichotomic at point $x_{0} \in \mathcal{X}$ if and only if there exist two functions $\varphi_{1}, \varphi_{2} \in \mathcal{F}$ and two projection families $\left\{\widetilde{Q}_{k}(t)\right\}_{t \geq 0}, k \in\{1,2\}$, compatible with $\xi$ such that following inequalities hold

$$
\begin{aligned}
& \left(\text { puet }_{0}\right) \varphi_{1}(t-s)\left\|\left[I-\widetilde{Q}_{1}(s)\right] v\right\| \leq\left\|\left[I-\widetilde{Q}_{1}(t+s)\right] \Psi\left(t, s, \psi\left(t, s, x_{0}\right)\right) v\right\| \\
& \left(\text { puet }_{0}^{\prime}\right) \varphi_{1}(t-s)\left\|\left[I-\widetilde{Q}_{2}(t+s)\right] \Psi\left(t, s, \psi\left(t, s, x_{0}\right)\right) v\right\| \leq\left\|\left[I-\widetilde{Q}_{2}(s)\right] v\right\| \\
& \left(\text { puet }_{1}\right)\left\|\Psi\left(t, s, \psi\left(t, s, x_{0}\right)\right) \widetilde{Q}_{1}(s) v\right\| \leq \varphi_{2}(t-s)\left\|\widetilde{Q}_{1}(s) v\right\| \\
& \left(\text { puet }_{2}\right)\left\|\widetilde{Q}_{2}(s) v\right\| \leq \varphi_{2}(t-s)\left\|\Psi\left(t, s, \psi\left(t, s, x_{0}\right)\right) \widetilde{Q}_{2}(s) v\right\|
\end{aligned}
$$

for all $(t, s) \in \mathcal{T}$ and all $v \in \mathcal{V}$. 
Proof. Necessity. The existence of functions $\varphi_{1}, \varphi_{2} \in \mathcal{F}$ and projections $\widetilde{Q}_{1}$ and $\widetilde{Q}_{2}$ is assured by Definition 2.11 if we consider

$$
\varphi_{1}(t)=N_{0}^{x_{0}} e^{-\nu_{0}^{x_{0}} t} \text { and } \varphi_{2}(t)=\max \left\{N_{1}^{x_{0}}, N_{2}^{x_{0}}\right\} e^{-\min \left\{\nu_{1}^{x_{0}}, \nu_{2}^{x_{0}}\right\} t}, t \geq 0
$$

respectively

$$
\widetilde{Q_{1}}(t)=\widetilde{P_{1}}(t), \widetilde{Q_{2}}(t)=\widetilde{P_{2}}(t), \quad t \geq 0
$$

and which verify the compatibility (see [9]) and $\left(\right.$ puet $\left._{0}\right)-\left(\right.$ puet $\left._{2}\right)$.

Sufficiency. We define

$$
\widetilde{P}_{0}(t)=I-\widetilde{Q}_{1}(t)-\widetilde{Q}_{2}(t), \widetilde{P}_{1}(t)=\widetilde{Q}_{1}(t), \widetilde{P}_{2}(t)=\widetilde{Q}_{2}(t), t \geq 0 .
$$

The compatibility of the projections families $\left\{\widetilde{P}_{k}(t)\right\}_{t \geq 0}, k \in\{0,1,2\}$ with $\xi$ is easy to verify (see [9]). By the properties of $\widetilde{Q}_{1}$ and $\widetilde{Q}_{2}$, it is true that

$$
\widetilde{P}_{0}(t)=\left[I-\widetilde{Q}_{1}(t)\right]\left[I-\widetilde{Q}_{2}(t)\right], \forall t \geq 0 .
$$

Let $x_{0} \in \mathcal{X}$ and $\varphi_{1} \in \mathcal{F}$. To prove the existence of $N_{0}^{x_{0}}$ and $\nu_{0}^{x_{0}}$ required by relation (2.10) we consider following inequalities which hold for all $\left(t, t_{0}\right) \in \mathcal{T}$ and all $v \in \mathcal{V}$

$$
\begin{gathered}
\left\|\widetilde{P}_{0}\left(t+t_{0}\right) \Psi\left(t, t_{0}, \psi\left(t, t_{0}, x_{0}\right)\right) v\right\| \geq \\
\geq \varphi_{1}(1)\left\|\Psi\left(t-1, t_{0}, \psi\left(t-1, t_{0}, x_{0}\right)\right)\left[I-\widetilde{Q}_{1}\left(t_{0}\right)\right]\left[I-\widetilde{Q}_{2}\left(t_{0}\right)\right] v\right\| \geq \ldots \geq \\
\geq \varphi_{1}^{\left[t-t_{0}\right]}(1)\left\|\Psi\left(t-\left[t-t_{0}\right], t_{0}, \psi\left(t_{0}, t_{0}, x_{0}\right)\right) \widetilde{P}_{0}\left(t_{0}\right) v\right\| \geq \\
\geq \varphi_{1}^{\left[t-t_{0}\right]}(\delta) \varphi_{1}(1)\left\|\Psi\left(t_{0}, t_{0}, \psi\left(t_{0}, t_{0}, x_{0}\right)\right) \widetilde{P}_{0}\left(t_{0}\right) v\right\|,
\end{gathered}
$$

where by $\left[t-t_{0}\right]$ we have denoted the integer part of the considered difference and the existence of $\delta>1$ is assured by the definition of the function set $\mathcal{F}$, such that $\varphi_{1}(\delta)<1$.

If we denote $N_{0}^{x_{0}}=\varphi_{1}(1)$ and $\nu_{0}^{x_{0}}=-\ln \varphi_{1}(\delta)$, the relation (2.10) is proved. Analogues techniques are used to prove inequality (2.11), as well as (2.12) and (2.13).

Some integral characterizations for the pointwise trichotomy are given by the next result, involving four compatible projector families, given by the next conditions.

Definition 3.2 Four projection families $\left\{\widetilde{R}_{k}(t)\right\}_{t \geq 0}$ where $\widetilde{R}_{k}: \mathbb{R}_{+} \rightarrow \mathfrak{B}(\mathcal{V})$, $k \in\{1,2,3,4\}$, are said to be compatible with a skew-evolution semiflow $\xi=(\psi, \Psi)$ at point $x \in \mathcal{X}$ if

$\left(c r_{1}\right) \widetilde{R}_{1}(t)+\widetilde{R}_{3}(t)=\widetilde{R}_{2}(t)+\widetilde{R}_{4}(t)=I$

$\left(c r_{2}\right) \widetilde{R}_{1}(t) \widetilde{R}_{2}(t)=\widetilde{R}_{2}(t) \widetilde{R}_{1}(t)=0$ and $\widetilde{R}_{3}(t) \widetilde{R}_{4}(t)=\widetilde{R}_{4}(t) \widetilde{R}_{3}(t)$ 


$$
\begin{aligned}
& \left(c r_{3}\right)\left\|\left[\widetilde{R}_{1}(t)+\widetilde{R}_{2}(t)\right] v\right\|^{2}=\left\|\widetilde{R}_{1}(t) v\right\|^{2}+\left\|\widetilde{R}_{2}(t) v\right\|^{2} \\
& \left(c r_{4}\right)\left\|\left[\widetilde{R}_{1}(t)+\widetilde{R}_{3}(t) \widetilde{R}_{4}(t)\right] v\right\|^{2}=\left\|\widetilde{R}_{1}(t) v\right\|^{2}+\left\|\widetilde{R}_{3}(t) \widetilde{R}_{4}(t) v\right\|^{2} \\
& \left(c r_{5}\right)\left\|\left[\widetilde{R}_{2}(t)+\widetilde{R}_{3}(t) \widetilde{R}_{4}(t)\right] v\right\|^{2}=\left\|\widetilde{R}_{2}(t) v\right\|^{2}+\left\|\widetilde{R}_{3}(t) \widetilde{R}_{4}(t) v\right\|^{2} \\
& \left(c r_{6}\right) \Psi\left(t, t_{0}, \psi\left(t, t_{0}, x\right)\right) \widetilde{R}_{k}\left(t_{0}\right) v=\widetilde{R}_{k}\left(t+t_{0}\right) \Psi\left(t, t_{0}, \psi\left(t, t_{0}, x\right)\right) v
\end{aligned}
$$

for all $t, t_{0} \geq 0, t \geq t_{0}$, for all $v \in \mathcal{V}$ and $k \in\{1,2,3,4\}$

Theorem 3.2 Let $\xi=(\psi, \Psi)$ be a skew-evolution semiflow such that for each $(x, v) \in \mathcal{Y}$ the mapping $s \rightarrow \Phi\left(s, t_{0}, x\right) v$ is continuous on $\left[t_{0}, \infty\right)$ and the mapping $s \rightarrow \Phi(t, s, x) v$ is continuous on $[0, t]$ and let us consider four projection families $\left\{\widetilde{R}_{k}(t)\right\}_{t \geq 0}, k \in\{1,2,3,4\}$, compatible with $\xi=(\psi, \Psi)$, with the property that there exist $s_{0}>t_{0} \geq 0$ and $c \in(0,1)$ such that

$$
\begin{aligned}
& \left\|\Psi\left(s_{0}, t_{0}, \psi\left(s_{0}, t_{0}, x\right)\right) \widetilde{R}_{1}\left(t_{0}\right) v\right\| \leq c\left\|\widetilde{R}_{1}\left(t_{0}\right) v\right\| \\
& \left\|\widetilde{R}_{2}\left(t_{0}\right) v\right\| \leq c\left\|\Psi\left(s_{0}, t_{0}, \psi\left(s_{0}, t_{0}, x\right)\right) \widetilde{R}_{2}\left(t_{0}\right) v\right\|
\end{aligned}
$$

for all $(x, v) \in \mathcal{Y}$.

Let $N, \widetilde{N}>1$ and $\omega, \widetilde{\omega}>0$ such that

$$
\begin{aligned}
& \left\|\Psi\left(t, t_{0}, x\right) \widetilde{R}_{i}\left(t_{0}\right) v\right\| \leq N e^{\omega(t-s)}\left\|\Psi\left(s, t_{0}, x\right) \widetilde{R}_{i}\left(t_{0}\right) v\right\|, i \in\{1,3\} \\
& \left\|\Psi\left(s, t_{0}, x\right) \widetilde{R}_{j}\left(t_{0}\right) v\right\| \leq \widetilde{N} e^{\widetilde{\omega}(t-s)}\left\|\Psi\left(t, t_{0}, x\right) \widetilde{R}_{j}\left(t_{0}\right) v\right\|, j \in\{2,4\}
\end{aligned}
$$

for all $(t, s),\left(s, t_{0}\right) \in \mathcal{T}$ and all $(x, v) \in \mathcal{Y}$.

Then the skew-product semiflow is pointwise uniformly exponentially trichotomic at point $x_{0} \in \mathcal{X}$ if and only if

(upet $_{0}$ ) there exist $M>0$ and $\alpha>0$ such that

$$
\int_{t_{0}}^{t} e^{-\alpha\left(\tau-t_{0}\right)}\left\|\Psi\left(\tau, t_{0}, \psi\left(\tau, t_{0}, x_{0}\right)\right) \widetilde{R}_{3}\left(t_{0}\right) v\right\| d \tau \leq M\left\|\widetilde{R}_{3}\left(t_{0}\right) v\right\|
$$

for all $\left(t, t_{0}\right) \in \mathcal{T}$ and all $v \in \mathcal{V}$

(upet $t_{0}^{\prime}$ ) there exist $M>0$ and $\alpha>0$ such that

$$
\int_{s}^{t} e^{\alpha(t-\tau)}\left\|\Psi\left(\tau, t_{0}, \psi\left(\tau, t_{0}, x_{0}\right)\right) \widetilde{R}_{4}\left(t_{0}\right) v\right\| d \tau \leq M\left\|\Psi\left(t, t_{0}, \psi\left(t, t_{0}, x_{0}\right)\right) \widetilde{R}_{4}\left(t_{0}\right) v\right\|
$$

for all $(t, s),\left(s, t_{0}\right) \in \mathcal{T}$ and all $v \in V$

(upet $_{1}$ ) there exists $M_{1}>0$ such that

$$
\int_{t_{0}}^{\infty}\left\|\Psi\left(\tau, t_{0}, \psi\left(\tau, t_{0}, x_{0}\right)\right) \widetilde{R}_{1}\left(t_{0}\right) v\right\| d \tau \leq M_{1}\left\|\widetilde{R}_{1}\left(t_{0}\right) v\right\|
$$

for all $t_{0} \in \mathbb{R}_{+}$and all $v \in V$ 
(upet $_{2}$ ) there exists $M_{2}>0$ such that

$$
\int_{0}^{t}\left\|\Psi\left(\tau, t_{0}, \psi\left(\tau, t_{0}, x_{0}\right)\right) \widetilde{R}_{2}\left(t_{0}\right) v\right\| d \tau \leq M_{2}\left\|\Psi\left(t, t_{0}, \psi\left(t, t_{0}, x_{0}\right)\right) \widetilde{R}_{2}\left(t_{0}\right) v\right\|
$$

for all $\left(t, t_{0}\right) \in \mathcal{T}$ and all $v \in V$.

Proof. Necessity. Considering the projection families $\left\{\widetilde{P}_{k}(t)\right\}_{t \geq 0}, k \in$ $\{0,1,2\}$, given as in Definition 2.11 we define

$$
\widetilde{R}_{1}(t)=\widetilde{P}_{1}(t), \widetilde{R}_{2}(t)=\widetilde{P}_{2}(t), \widetilde{R}_{3}(t)=I-\widetilde{P}_{1}(t), \widetilde{R}_{4}(t)=I-\widetilde{P}_{2}(t), t \geq 0 .
$$

We also have $\widetilde{R}_{3}(t) \widetilde{R}_{4}(t)=\widetilde{R}_{4}(t) \widetilde{R}_{3}(t)=\widetilde{P}_{0}(t), t \geq 0$. The compatibility is easy to verify, as in [9]. We denote by $N^{x_{0}}=\max \left\{N_{0}^{x_{0}}, N_{1}^{x_{0}}, N_{2}^{x_{0}}\right\}$ and $\nu_{1}^{x_{0}}, \nu_{2}^{x_{0}}, \nu_{0}^{x_{0}}$ the characteristics of the pointwise uniformly exponentially trichotomic skew-evolution semiflow $\xi$, given by Definition 2.11,

To prove $\left(\right.$ upet $\left._{1}\right)$, we consider $M_{1}=N^{x_{0}}\left(\nu_{1}^{x_{0}}\right)^{-1}$. For $\left(\right.$ upet $\left._{2}\right)$ let us denote $M_{2}=N^{x_{0}}\left(\nu_{2}^{x_{0}}\right)^{-1}$. We obtain $\left(\right.$ upet $\left._{0}\right)$ and (upet ${ }_{0}^{\prime}$ ) if we consider $\alpha=2 \nu_{0}^{x_{0}}$ and $M=N^{x_{0}}\left(\nu_{0}^{x_{0}}\right)^{-1}$.

Sufficiency. We define

$$
\widetilde{P}_{1}(t)=\widetilde{R}_{1}(t), \widetilde{P}_{2}(t)=\widetilde{R}_{2}(t), \widetilde{P}_{0}(t)=\widetilde{R}_{3}(t) \widetilde{R}_{4}(t), t \geq 0 .
$$

The compatibility is obtained from the properties of the projection families $\left\{\widetilde{R}_{k}(t)\right\}_{t \geq 0}, k \in\{1,2,3,4\}$.

Relation (upet $t_{0}$ ) defines the integral stability for the skew-evolution flow given by $\xi_{\alpha}=\left(\psi, \Psi_{\alpha}\right)$, where $\Psi_{\alpha}(t, s, x)=e^{-\alpha(t-s)} \Psi(t, s, x),(t, s) \in \mathcal{T}, x \in$ $\mathcal{X}$. As relation (3.3) hold, then according to a result that characterize the stability obtained in [12] it follows that $\xi_{\alpha}$ is uniformly exponentially stable, which assures the existence of some constants $K>0$ and $\beta>0$ such that we obtain successively for all $(t, s),\left(s, t_{0}\right) \in \mathcal{T}$ and all $(x, v) \in \mathcal{Y}$

$$
\begin{gathered}
\left.\left.\| \Psi\left(t, t_{0}, \psi\left(t, t_{0}, x\right)\right) \widetilde{R}_{3}\left(t_{0}\right) v\right)\left\|=e^{\alpha\left(t-t_{0}\right)}\right\| \Psi_{\alpha}\left(t, t_{0}, \psi\left(t, t_{0}, x\right)\right) \widetilde{R}_{3}\left(t_{0}\right) v\right) \| \leq \\
\left.\leq K e^{-\beta(t-s)} e^{\alpha\left(t-t_{0}\right)} \| \Psi_{\alpha}\left(s, t_{0}, \psi\left(s, t_{0}, x\right)\right) \widetilde{R}_{3}\left(t_{0}\right) v\right) \|= \\
\left.=K e^{(\alpha-\beta)(t-s)} \| \Psi\left(s, t_{0}, \psi\left(s, t_{0}, x\right)\right) \widetilde{R}_{3}\left(t_{0}\right) v\right) \|
\end{gathered}
$$

and, if we define

$$
\nu_{0}^{x_{0}}=\left\{\begin{array}{cc}
\alpha-\beta, & \text { if } \alpha>\beta \\
1, & \text { if } \alpha \leq \beta
\end{array}\right.
$$

then (2.10) is proved. By an analogue proof is obtained (2.11) from (upet $\left.{ }_{0}^{\prime}\right)$.

As in [6], from the property that there exist $t_{0}>0$ and $c \in(0,1)$ such that relation (3.1) hold it follows that

$$
\left\|\Psi\left(t, t_{0}, \psi\left(t, t_{0}, x\right)\right) \widetilde{R}_{1}\left(t_{0}\right) v\right\| \leq N_{1}^{x_{0}} e^{\nu_{1}^{x_{0}}}\left\|\widetilde{R}_{1}\left(t_{0}\right) v\right\|
$$


for all $\left(t, t_{0}\right) \in \mathcal{T}$ and all $(x, v) \in \mathcal{Y}$, where

$$
\nu_{1}^{x_{0}}=\frac{\ln c}{t_{0}}, N_{1}^{x_{0}}=N e^{\left(\omega+\nu_{1}^{x_{0}}\right) t_{0}}
$$

and constants $N$ and $\omega$ are given by (3.3).

From the fact that there exist $t_{0}>0$ and $c \in(0,1)$ such that inequality (3.2) holds it follows that

$$
\left\|\widetilde{R}_{2}\left(t_{0}\right) v\right\| \leq N_{2}^{x_{0}} e^{-\nu_{2}^{x_{0}} t}\left\|\Psi\left(t, t_{0}, \psi\left(t, t_{0}, x\right)\right) \widetilde{R}_{2}\left(t_{0}\right) v\right\|
$$

for all $\left(t, t_{0}\right) \in \mathcal{T}$ and all $(x, v) \in \mathcal{Y}$, where

$$
\nu_{2}^{x_{0}}=-\frac{\ln c}{t_{0}}, N_{2}^{x_{0}}=\widetilde{N} e^{\widetilde{\omega} t_{0}} .
$$

and constants $\widetilde{N}$ and $\widetilde{\omega}$ are given by (3.4).

Hence, the pointwise uniform exponential trichotomy for the skew-evolution semiflow $\xi$ is proved.

\section{References}

[1] S.N. Chow, H. LeIVA, Existence and roughness of the exponential dichotomy for linear skew-product semiflows in Banach spaces, J. Differential Equations 120 (1995) 429-477.

[2] S. Elaydi, O. HajeK, Exponential trichotomy of differential systems, J. Math. Anal. Appl. 129 (1988) 362-374.

[3] Y. Latushinin, S. Montgomery-Smith, T. RAndolph, Evolutionary semigroups and dichotomy of linear skew-product flows on locally compact spaces with Banach fibers, J. Differential Equations 125 (1996) 73-116.

[4] Y. Latushin, R. Schnaubelt, Exponential dichotomy of cocycles, evolution semigroups and translation algebras, J. Differential Equations 159 No.2 (1999) 321-369.

[5] M. Megan, A.L. Sasu, B. Sasu, Stabilizability and controllability of systems associated to linear skew-product semiflows, Rev. Mat. Complut. 15 Num.2 (2002) 599-618.

[6] M. Megan, A.L. Sasu, B. Sasu, Exponential stability and exponential instability for linear skew-product flows, Math. Bohem. 129 No.3 (2004) 225-243.

[7] M. Megan, A.L. Sasu, B. SAsu, Perron conditions for pointwise and global exponential dichotomy of linear skew-product flows, Integral Equations and Operator Theory 50 (2004) 489-504.

[8] M. Megan, C. StoicA, On uniform exponential trichotomy of evolution operators in Banach spaces, accepted for publishing in Integral Equations and Operator Theory.

[9] M. Megan, C. Stoica, Equivalent definitions for uniform exponential trichotomy of evolution operators in Banach spaces, accepted for publishing in J. Operator Theory.

[10] M. Megan, C. Stoica, L. Buliga, On asymptotic behaviors for linear skewevolution semiflows in Banach spaces, Carpathian J. Math. 23 (2007) 117-125.

[11] R.J. SACKeR, G.R. SELl, Existence of dichotomies and invariant splittings for linear differential systems I, II, III, J. Differential Equations 15, 22 (1974, 1976) 429-458, 478-496, 497-522. 
[12] C. Stoica, M. Megan, On asymptotic behaviors of evolution operators in Banach spaces, Ser. Preprint. Mat., Univ. Timişoara 5 (2006) 1-22.

\section{Codruţa Stoica}

Institut de Mathématiques

Université Bordeaux 1

France

e-mail: codruta.stoica@math.u-bordeaux1.fr 